\title{
E.coli JM83 Damages Mucosal Barrier Inducing Hirschsprung-Associated Enterocolitis via Activated TLR4 / NF-kB / P-p38 Signaling
}

\section{Zebing Zheng ( $\square$ zebing1988@sina.com )}

Soochow University Affiliated Children's Hospital: Children's Hospital of Soochow University https://orcid.org/0000-0003-3392-7104

\section{Mingjuan Gao}

Affiliated Hospital of Zunyi Medical University

Chengyan Tang

Affiliated Hospital of Zunyi Medical University

Lu Huang

Affiliated Hospital of Zunyi Medical University

\section{Yuan Gong}

Affiliated Hospital of Zunyi Medical University

Yuanmei Liu

Affiliated Hospital of Zunyi Medical University

Jian Wang

Soochow University Affiliated Children's Hospital: Children's Hospital of Soochow University

\section{Research Article}

Keywords: Hirschsprung-associated enterocolitis, TLR4, mucosal barrier,Ednrb

Posted Date: November 8th, 2021

DOI: https://doi.org/10.21203/rs.3.rs-1047222/v1

License: (1) (i) This work is licensed under a Creative Commons Attribution 4.0 International License. Read Full License 


\section{Abstract \\ Purpose}

Hirschsprung-associated enterocolitis (HAEC) is characterized by intestinal mucosal damage and unbalance of intestinal microbiota. Recent studies have shown that the TLR4/NF-KB/p-p38 signaling in the intestine is of great importance to intestinal mucosal integrity. This study aimed to investigate the role of TLR4/NF-KB/p-p38 signaling in the pathogenesis of HAEC in Escherichia coli (E. coli) JM83 infected Endothelin receptor $\mathrm{B}(E d n r b)^{-/-}$mice.

\section{Methods}

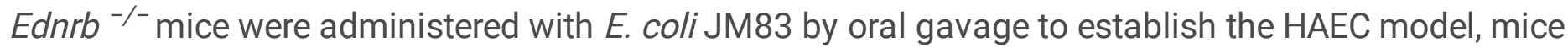
were randomly divided into WT group, $E d n r b^{-/-}$group and $E d n r b^{-/-}+E$. coli JM83 group. The role of TLR4/NF-kB/p-p38 signaling was evaluated by vivo study.

\section{Results}

The activation of the TLR4/NF-KB/p-p38 signaling induced by E. coli JM83 caused HAEC in Ednrb ${ }^{-/-}$ mice, which was evidenced by a significantly increased expression of TNF-a, TGF- $\beta$ and IL-10, decreased density of F-actin protein. While TLR4 knockdown improved the degree of enterocolitis and attenuated the expression of IL-10, TNF-a, TGF- $\beta$ and increased the density of F-actin protein in $E d n r b^{-/-}$mice after $E$. coli infection.

\section{Conclusions}

These results indicate that $E$. coli JM83 activates TLR4/NF-KB/p-p38 signaling to promote the development of HAEC. However, inhibition of this signaling may be benefit to the treatment and prevention of HAEC.

\section{Introduction}

Hirschsprung-associated enterocolitis (HAEC) is the most common complication of Hirschsprung disease (HSCR) [1, 2], which occurred in the preoperative or postoperative stages even after definitive pull-through surgery. Accumulating clinical evidence suggests that abnormalities in the intestinal microbiome, impaired intestinal mucosal barrier function, an altered systemic immune system and bacterial translocation are all possible causes of HAEC [3-5]. Intestinal tract is the most active immune organ in human body, which is constantly challenged by a large number of antigens. Clostridium difficile, Escherichia coli (E. coli) and certain viruses have been suggested as causative organisms of enterocolitis 
development [6]. The method in which gut microbes influence the mucosal barrier and the development of pathogenic bacteria-mediated intestinal inflammatory disease remain unclear, but the deficiency of intestinal repair of inflammatory-driven injury may play an important role in the pathogenesis of HAEC.

Previous studies reported that the disorder of TLR4 signaling transduction led to the uncontrolled colitis, which was associated with the loss of mucosal integrity, development of ulcerations, colonic bleeding [7], these clinical features were in consistent with HAEC patients. Emerging evidence shows that TLR4 expression is increased in several intestinal inflammatory diseases, including inflammatory bowel colitis [8] and necrotizing enterocolitis [9]. When TLR4 is engaged by its ligands, the downstream signaling pathways, including the nuclear factor-KB (NF-KB) and MAPK p38 (p-p38) pathways, are activated; this activation is essential for the initiation of an inflammatory response by promoting and/or modulating the transcription and translation of inflammation-related genes, such as interleukin-6 (IL-6), tumor necrosis factor- $\alpha$ (TNF- $a$ ) and IL-1 $\beta$ [10]. One literature reveals that the inhibition of NF-KB can protect the colonic epithelium from damage and/or promote epithelial repair [11, 12].

We hypothesized that the pathogenic organism E. coli JM83 promoted HAEC development through TLR4/NF-kB/p-p38 signaling, influencing intestinal mucosal barrier integrity. In this study, it was investigated whether TLR4/NF-KB/p-p38 signaling participated in the pathogenesis of HAEC during the invasive infection of $E d n r b^{-/-}$mice with E. coli JM83 (used as an HAEC model).

\section{Materials And Methods}

\section{Animals}

Wild-type (WT) $(n=20)$ and Ednrb flex3/flex3 $(n=8)$ mice were purchased from the Institute of Model Animals of Wuhan University (Wuhan, China). Endothelin receptor B-null (Ednrb flex3/flex3) was used on the C57/B6J

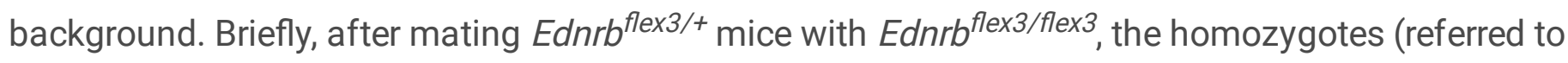
herein as $E d n r b^{-/-}$) were easily distinguished from the WT and heterozygote littermates (referred to herein as $E d n r b^{+/+}$and $E d n r b^{+/-}$, respectively) by the white fur color and gradually enlarging abdomens (due to absence of ganglion cells at the end of rectum). Ednrb ${ }^{+/+}$and $E d n r b^{+/-}$were normal phenotypes and didn't develope aganglionosis. Therefore, $20 \mathrm{Ednrb}^{+/+}$or $E d n r b^{+/-}$animals were randomly assigned to WT group. Total $20 \mathrm{Ednrb}^{-/-}$animals were obtained in this study which displayed distal colonic aganglionosis involving 5-10 mm of the colon. Naive mice were defined as WT and Ednrb ${ }^{-/-}$mice without any intervention. According to the manufacturer's protocols. Briefly, after anesthetized with ether (2-4 \%), a $2 \mathrm{~mm}$ diameter anal canal was inserted into the anus of the experimental mice, the 5 mice were randomly selected form WT and $E d n r b^{-/-}$groups were transfected with small interfering RNA (siRNA) duplexes (100 nM) into colon targeting TLR4. TLR4 forward, sense strand: 5'- UUCGAGACUGGACAAGCC -3 ' and an antisense strand: 5'- UGGCUUGUCCAGUCACGA -3' (20 nM; Guangzhou RiboBio Co., Ltd.) to generate WT + TLR4 siRNA ( $\mathrm{n}=5)$ and $E d n r b^{-/-}+T L R 4$ siRNA(n=5) mice groups [13]. The knockdown of TLR4 gene was confirmed at protein level by western blot. The whole experiment lasted for 40 days after 
mice birth, all experiments involving animals were performed in a specific pathogen-free environment, in accordance with the Zunyi Medical University (Zunyi, China) Guidelines for Animal Care. Animals were kept under a 12-h light/dark cycle with access to food and water.

\section{Ethic Statements}

All animal experimental protocols were complied with the Guide for the Care and Use of Laboratory Animals published by the Zunyi Medical University (Zunyi, China). The present study was approved by the Institutional Animal Research Committee of Zunyi Medical University (approval no. IACUC-20191025028; Guizhou, China).

\section{Bacterial Strains And Infection Of Mice}

WT, WT + TLR4 siRNA, Ednrb ${ }^{-/-}$, and $E d n r b^{-/-}+T L R 4$ siRNA mice were infected with $0.1 \mathrm{ml}$ Lb containing $1 \times 10^{9}$ cfu E. coli JM83 (Jackson Laboratory) by oral gavage to establish the WT + E. coli $(\mathrm{n}=5), \mathrm{WT}+$ $T L R 4$ siRNA + E. coli $(\mathrm{n}=5), E d n r b^{-/-}+E$. coli $(\mathrm{n}=5)$, and $E d n r b^{-/-}+T L R 4$ siRNA + E. coli $(\mathrm{n}=5)$ groups. The $E$. coli JM83 were cultured on trypsin soybean agar plates supplemented with $5 \%$ sheep blood (cat no. 21374; BD Biosciences), which was in turn supplemented with $0.2 \%$ yeast extract (cat. no. 843; Merck $\mathrm{KGaA}$ ) at $37^{\circ} \mathrm{C}$ with $5 \% \mathrm{CO}_{2}$ (durations indicated in individual experiments).

\section{Establishment Of Haec Model}

The colon samples from Ednrb ${ }^{-/}+E$. coli mice were stored in $4 \%$ buffered formalin and embedded with paraffin for subsequent hematoxylin and eosin and immunofluorescence staining analysis to verify the HAEC model. Inflammatory cell infiltration of the crypts (cryptitis and crypt abscesses) was lighter in HAEC mice than in human beings. The severity of HAEC was evaluated according to the modified grading system reported by Porokuokka et al [14] to recently reflect HAEC mice epithelial pathology. Parts of the small bowel (jejunum and ileum) or large bowel (cecum and colon) were excised by separation from the mesentery to prepare a single intestinal cell suspension.

\section{Hematoxylin \&eosin (He) And Immunohistochemistry (Ihc)}

The colon sections were removed following euthanasia and cut to a thickness of $3 \mathrm{~mm}$, stained with $\mathrm{HE}$ and imaged by light microscopy (Nikon Corporation, magnification, $\mathrm{x} 20$ ). The stained section assigned an inflammation score in a blinded manner, as previously described [14]. TLR4 protein expression levels were detected by $\mathrm{IHC}$. The IHC sections were incubated pass the night at $4^{\circ} \mathrm{C}$ in primary antibody solution containing anti-human TLR4 antibody (cat. no. A0456; dilution, $2 \mu \mathrm{g} / \mathrm{l}, 1: 200$; OriGene Technologies, Inc.) and a biotin-streptavidin HRP detection system for $12 \mathrm{~h}$, followed by detection with HRP-conjugated goat 
anti-rabbit IgG secondary antibody (cat. no. 2019629, 1:200; Beijing Zhongshan Golden Bridge Biotechnology Co., Ltd.). Negative controls were treated with PBS instead of primary antibodies. All sections were observed by an optical microscope (OLYMPUS BH-2; Olympus Corporation, magnification, $\mathrm{x} 100)$.

\section{Immunofluorescence}

For immunofluorescence staining, colon tissue was separated from mice. Colon tissue was fixed in PBS $/ 4 \%$ PFA and $10 \%$ sucrose solution at $4^{\circ} \mathrm{C}$ for $1 \mathrm{~h}$, followed by overnight cryoprotection in PBS/30\% sucrose (cat no. 7124; Merck KGaA) for 3 days at $4^{\circ} \mathrm{C}$. Tissue sections were cut into $20-\mathrm{mm}$ sections using a Leica Cryostat Microtome and blocked using a Streptavidin/Biotin Blocking kit (Vector Laboratories, Inc.) and stored at $-80^{\circ} \mathrm{C}$ until processing. Colon tissue samples were stained with either mouse or rabbit anti-F-actin (cat. no.8927, 1:2,000; Merck KGaA), with the addition of DAPI (cat no. 6982; BioLegend, Inc.). Sections were mounted in a FV1000 laser-scanning confocal microscope (Olympus Corporation, magnification, $\mathrm{x} 20$ ).

\section{Western Blot Analysis}

Western blot analysis was performed following the manufacturer's protocol and concentration were measured by the Enhanced Bicinchoninic Acid Protein Assay kit (Beyotime Institute of Biotechnology, Jiangsu, China). Forty $\mu$ l Proteins were loaded in each well. extracts $(120 \mu l)$ were mixed with SDS-PAGE (Beyotime Institute of Biotechnology), heated at $90^{\circ} \mathrm{C}$ for $5 \mathrm{~min}$. And then transferred to polyvinylidene fluoride membranes. The membranes were blocked with $5 \%$ fat-free milk in Tris-buffered saline for $45 \mathrm{~min}$ at room temperature. The membrane was the wash with TBST, and then incubated with primary rabbit anti-mouse antibodies anti-TLR4 (1:200, cat. no. ab22048), anti-NF-kBp65 (1:200, cat. no. ab16502), antiphosphorylated (p)-p38 (1:200, cat. no. ab31828) (all Abcam), anti-p38 (1:200, cat. no. ab2749), and antiGAPDH (1:200, cat. no. 60004) and mouse anti-rabbit IgG HRP-conjugated (cat. no. sc-2357) (both Beyotime Institute of Biotechnology) at $4^{\circ} \mathrm{C}$ for $16 \mathrm{~h}$. The membranes were washed with TBST three times and subsequently incubated with secondary antibodies [TLR4 (1:4,000, cat. no. ab23548), NF-kB (1:4,000, cat. no. ab17893), p-p38(1:4,000, cat. no. ab34620), p38(1:4,000, cat. no. ab28495)] for $2 \mathrm{~h}$ at room temperature.

\section{Quantitative Pcr (Qpcr)}

For qPCR, total RNA was isolated from colon tissue and macrophages using TRIzol® reagent (Invitrogen; Thermo Fisher Scientific, Inc.) according to the manufacture's instructions. The NanoDrop 2000 spectrophotometer (Thermo Fisher Scientific, Inc.) was used for qRNA concentration analysis. qPCR amplification was subsequently performed on an ABI 7900HT Real-Time PCR Detection system (Applied Biosystems; Thermo Fisher Scientific, Inc.) to measure the expression of mRNA. The specific primers 
used were as (Table 1). The qPCR cycle program was: initial denaturation $50^{\circ} \mathrm{C}$ for $2 \mathrm{~min}, 95^{\circ} \mathrm{C}$ for $10 \mathrm{~min}$, followed by 40 cycles of $95^{\circ} \mathrm{C}$ for $15 \mathrm{sec}$ and $60^{\circ} \mathrm{C}$ for $60 \mathrm{sec}$. Data analysis was calculated by the $2^{-\Delta \Delta \mathrm{Cq}}$ method.

Table 1

Primer sequences used for quantitative PCR.

\begin{tabular}{|lll|}
\hline Gene name & Primer sequences $\left(\mathbf{5}^{\mathbf{}^{\prime}} \mathbf{3}^{\prime} \mathbf{)}\right.$ & \\
\cline { 2 - 3 } & Forward & Reverse \\
\hline TLR4 & AGCAGAGGAGAAAGCATCTATGATGC & GGTTTAGGCCCCAGAGTTTTTCTCC \\
\hline p38 & CGACTTGCTGGAGAAGATGC & TCCATCTCTTCTTGGTCAAGG \\
\hline NF-KB & ACATCGTGGTCGGCTTCG & GGGTCACCAGGTACACGTCATT \\
\hline GAPDH & GACGGCCGCATCTTCTTGT & CACACCGACCTTCACCATTTT \\
\hline
\end{tabular}

\section{ELISA}

The levels of IL-10, TNF- $\alpha$ and transforming growth factor $\beta$ (TGF- $\beta$ ) in colon tissue and cell culture supernatants were measured using commercially available [IL-10 (cat. no.H009), TNF- a (cat. no.H052), TGF- $\beta$ (cat. no.H034) ELISA kits, according to the manufacturer's instructions (all Nanjing Jiancheng Bioengineering Inc.).

\section{Statistical analysis}

SPSS statistical software package (version 19.0; IBM Crop., Armonk, NY, USA) and Graphpad Prism statistical software package (version 8.0; Graphpad software Inc., La Jolla, CA, USA) were used for statistical analysis. Normally distributed data were presented as the mean \pm standard deviation (SD). And statistical analysis were performed using Student's t-test and one-way ANOVA test. Statistical comparisons of two or more groups with two independent variables were analyzed by two-way ANOVA and a Bonferroni's post-hoc test. $P$ value $<0.05$ was considered statistically significant.

\section{Results}

\section{TLR4/NF-kB/p-p38 signaling participates in the pathogenesis of HAEC.}

We employed the endothelin receptor B targeted-null mouse (Figure 1a, 1b) to exhibit the pathological features of HSCR, such as aganglionosis in the rectum and distal colon, as well as enterocolitis [13]. First, WT and Ednrb ${ }^{-/-}$mice were infected with E. coli JM83, and inflammation scores in the colon were histologically assessed. As shown in Figure 2, naive $\mathrm{Ednrb}^{-/-}$mice developed enterocolitis spontaneously at 5 weeks (Figure 2c), whereas $E d n r^{-/-}$mice infected with E. coli succumbed to lethal enterocolitis at 3 weeks (Figure 2d). By contrast, WT mice infected with E. coli only developed mild inflammation in the colon (Figure 2b). 
Immunohistochemistry revealed a strong brown expression of TLR4 in the colon of Ednrb ${ }^{-/-}$(Figure 2g) and $E$. coli-infected $E d n r b^{-/-}$mice (Figure 2h), compared with WT (Figure 2e) and E. coli-infected WT mice (Figure 2f). Western blot analysis and qPCR further confirmed that the protein and mRNA levels of TLR4, NF-KB and p-p38/p38 were significantly increased in Ednrb ${ }^{-/-}$and E. coli-infected Ednrb ${ }^{-/-}$mice $(\mathrm{P}<0.05$ vs. WT and $E$. coli-infected WT mice), with no significant difference identified between $E d n r b^{-/-}$and $E$. coli-infected $E_{n n r b^{-/-}}$mice ( $P>0.05$; Figure $1 \mathrm{c}, 1 \mathrm{~d}, 1 \mathrm{e}$ ). In addition, the expression of TNF- $\alpha$, TGF- $\beta$ and IL10 were increased in $E d n r b^{-/-}$and E. coli-infected $E d n r b^{-/-}$mice, compared with WT and E. coli-infected WT mice ( $\mathrm{P}<0.05$; Figure 1f). Of note, E. coli-infected Ednrb- ${ }^{-/-}$mice exhibited markedly higher TNF-a, TGF$\beta$ and IL-10 levels than Ednrb ${ }^{-/-}$mice $(P<0.05$; Figure 1f).

\section{TLR4 knockdown reverses intestinal inflammation}

To determine the importance of TLR4/NF-KB/p-p38signaling in E. coli JM83 infection-induced HAEC, the severity of enterocolitis and cytoskeletal F-actin expression, a critical structure for preserving the integrity of the epithelial barrier, was assessed by TLR4 knockdown in WT and Ednrb ${ }^{-/}$mice. A marked interruption in mucosal structures was observed, and large numbers of inflammatory cells and abscesses were found to have infiltrated the mucosa and sub-mucosa upon E. coli infection in Ednrb ${ }^{-/}$mice (Figure 3c), whereas the severity of $E$. coli infection-induced enterocolitis was markedly alleviated in $E d n r b^{-/}$mice following TLR4 knockdown (Figure 3d). In addition, the mild degree of inflammation in the colon observed in E. coliinfected WT mice was almost completely reversed by TLR4 knockdown (Figure 3b).

F-actin expression was increased in the cytoplasm of intestinal epithelial cells, alongside increased tight junction integrity in the intestinal mucosal barrier in both WT and TLR4 siRNA-transfected WT mice 3 weeks after E. coli infection (Figure 4a, 4b). By contrast, Ednrb ${ }^{-/}$mice exhibited a substantially decreased density of F-actin protein and severely disordered tight junction structures in response to $E$. coli infection (Figure 4c). Of note, TLR4 knockdown in Ednrb ${ }^{-/}$mice gradually increased the density of F-actin and partly reversed tight junction integrity (Figure $4 d$ ).

\section{TLR4 knockdown reduces inflammatory reaction in Ednrb ${ }^{-/}$mice and suppresses downstream TLR4/NF- KB/p-p38 signaling}

To ascertain whether TLR4/NF-kB/p-p38 signaling was critical to the progression of HAEC, TLR4mediated downstream pathways and inflammatory cytokines were examined following TLR4 knockdown in WT and $E d n r b^{-/-}$mice after E. coli infection. The activation of downstream TLR4/NF-KB/p-p38 signaling pathways, including NF-kB and p-p38/p38, were further examined. As shown in Figure $5 a$, the transfection of both WT and Ednrb ${ }^{-/-}$mice with TLR4 siRNA efficiently knocked down TLR4 protein expression. Of note, TLR4 knockdown in Ednrb ${ }^{-/-}$mice strongly suppressed NF-kB and p-p38 expression

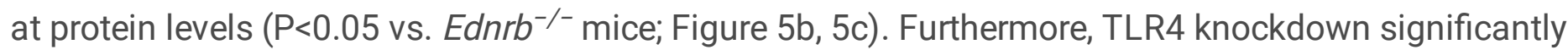
attenuated TNF-a, TGF- $\beta$ and IL-10 expression in Ednrb ${ }^{-/-}$mice ( $\mathrm{P}<0.05$ vs. Ednrb ${ }^{-/-}$mice and WT mice, Figure 5d). 


\section{Discussion}

HAEC can occur at any time before, during and after endorectal pull-through surgery, which is the definitive procedure for HSCR [15]. There exists a wide variation in the reported incidence of HAEC, which occurs in $2-33 \%$ of patients with common type and $50 \%$ of patients with long type HSCR. Clinically, HAEC is characterized by discomfort, decreased appetite, abdominal distention, loose foul-smelling stools, fever and sepsis [16]. Previous studies tended to believe that the postoperative HAEC were related to surgical factors, such as anastomotic stricture or leak, and bowel obstructions $[1,4,17-20]$. At present, many results have indicated that the pathogenesis of HAEC are related to mucosal barrier, intestinal microbiota, and immune function [21-23]. At least partly due to the widely use of $E d n r b^{-/-}$animal in HAEC researches, the order in which these HAEC-related etiology features change is gradually understood, which has promoted improvements in the treatment and prevention of HAEC [24]. A previous multicenter study has identified that the HAEC patients showed reduced abundance of the phyla Firmicutes and increased Bacteroidetes and Proteobacteria, by comparing the bacterial microbiome composition of children with HSCR to those who had a history of HAEC [25]. These results strongly indicate a dysequilibrium in the gut microbial ecosystem of HAEC patients, such that the dominance of bacteria ( $E$. coli) predisposes patients to the development of HAEC.

In this study, E. coli JM83 was used as the pathogenic bacteria to infect the intestine of $E d n r b^{-/-}$mice to establish an HAEC mouse model. A previous study reported that $E d n r b^{-/-}$mice developed HAEC on postnatal days $24-26$ and $100 \%$ mortality was recorded by day 28 after birth [26]. Clinical histopathologic features of HAEC patients mainly include the colon crypt dilatation, mucin retention, enterocyte adherence of bacteria, epithelial damage, leukocyte infiltration, ulceration, and in the terminal stages, transmural necrosis and perforation $[27,28]$. In our study, the histopathological results showed that a number of inflammatory cells infiltrated the mucosa and submucosa of the intestinal wall, even abscesses were observed in E. coli JM83-infected $E d n r b^{-/-}$mice, which was in accordance with the human manifestation. $E d n r b^{-/-}$mice developed HAEC 3 weeks after E. coli JM83 infection, with a few mice dying of abdominal distention, diarrhea and dehydration.

A study showed the passive transport of mucosal barrier in Ednrb ${ }^{-/-}$mice, the E. colitransport was significantly reduced in proximal colon rather than in the distal colon [29]. Previous studies showed that the dysfunction of intestinal epithelial contributed to the reduction in expression and changes in distributions of these F-actin, influencing the barrier function and increasing permeability[30, 31]. The expression of the F-actin gradually decreased with activating TLR4/NF-KB/p-p38 signaling, and therefore eventually led to intestinal mucosal damage. Studies reported that TLR4 activated by bacteria may be a major mediator of activating intestinal mucosal immunity, advancement of intestinal inflammation and immune response promotion[32]. Therefore, our results match the fact that HAEC may happen even after the postoperative pull-through surgery.

TLR4/NF-KB/p-p38 signaling pathway is transmitted through adaptor proteins, and signaling through MyD88 may be necessary to drive phagocytosis $[8,33,34]$. Studies have shown that the main function of 
TLR4 signaling in macrophages is to induce inflammatory response and protect host from pathogenic bacteria [35]. Whereas, the In vivo experiments of the present study revealed that the TLR4 protein receptors accumulated in the colon for 3 weeks following stimulation with E. coli JM83. TLR4 stimulated NF-KB through MyD88 in a mice model, and the level of NF-KB and p-p38/p38 was increased in the colon wall following stimulation with E. coli JM83. Likewise, NF-KB induced TNF- $\alpha$ and TGF- $\beta$ increased with the degree of enterocolitis in $E d n r b^{-/-}$mice, and therefore eventually led to intestinal mucosal damage. The above results indicate that TLR4/NF-KB/p-p38 signaling pathway plays a central role in the initiation of innate cellular immune responses, in the development of subsequent adaptive immune responses to invading bacterial infection, and eventually promoting intestinal mucosal tissue damage in HAEC. This process is consistent with the pathogenesis of IBD in past adult studies [36]. By contrast, the mucosal barrier integrity was maintained without the development of enterocolitis following TLR4 knockdown. Former studies have indicated that enhanced TLR4 expression is related to mortality in sepsis model [37].

However, siRNA-transfected TLR4/NF-kB/p-p38 signaling could reverse the inflammatory effects caused by $E$. coli infection, indicating that TLR4/NF-KB/p-p38 signaling plays a central role in maintaining the balance of gut homeostasis during the pathogenesis of HAEC. Under certain conditions, this downregulation of TLR4 signaling would ameliorate the degree of immune-mediated enterocolitis, provides a new idea for the treatment and prevention of HAEC.

In conclusion, the present study highlights the response of the intestinal mucosal barrier to HAEC induced by the pathogenic bacteria of E. coli. In addition, the activation of TLR4/NF-KB/p-p38 signaling in Ednrb-/ mice by $E$. coli JM83 lead to the development of inflammation, which is shown to be an underlying mechanism. Furthermore, inhibition of TLR4/NF-KB/p-p38 signaling may be benefit to the treatment and prevention of HAEC, contributing to the improvement of intestinal mucosal integrity.

\section{Abbreviations}

HAEC, Hirschsprung-associated enterocolitis; HSCR, Hirschsprung disease; TLR4, Toll-like receptor 4; WT, wild-type mice; $E d n r b$, endothelin receptor B; ENS, enteric nervous system; IBD, inflammatory bowel colitis; NF-kB, nuclear factor-kB; p-p38, MAPK p38; IL-10, interleukin-10; TNF- $\bigotimes$, tumor necrosis factor- $\varangle ;$ TGF- $\beta$, transforming growth factor $\beta$.

\section{Declarations}

\section{Acknowledgements}

Not applicable.

\section{Funding}

This work was supported by the Joint Fund of the Department of Guizhou Science and Technology of China (grant nos. 20177100 and 20204Y005). 


\section{Conflicts of interest}

The authors declares that there have no competing interest

\section{References}

1. Le-Nguyen A, Righini-Grunder F, Piche N, Faure C, Aspirot A (2019) Factors influencing the incidence of Hirschsprung associated enterocolitis (HAEC). J Pediatr Surg 5 (54): 959-963. https://doi.org/10.1016/j.jpedsurg.2019.01.026

2. Nakamura H, Lim T, Puri P (2018) Probiotics for the prevention of Hirschsprung-associated enterocolitis: a systematic review and meta-analysis. Pediatr Surg Int 2 (34): 189-193. https://doi.org/10.1007/s00383-017-4188-y

3. Austin KM (2012) The pathogenesis of Hirschsprung's disease-associated enterocolitis. Seminars in Pediatric Surgery 4 (21): 319-327. https://doi.org/10.1053/j.sempedsurg.2012.07.006

4. Cheng S, Wang J, Pan W, et al (2017) Pathologically assessed grade of Hirschsprung-associated enterocolitis in resected colon in children with Hirschsprung's disease predicts postoperative bowel function. J Pediatr Surg 11 (52): 1776-1781. https://doi.org/10.1016/j.jpedsurg.2017.03.056

5. Frykman PK, Nordenskjold A, Kawaguchi A, et al (2015) Characterization of Bacterial and Fungal Microbiome in Children with Hirschsprung Disease with and without a History of Enterocolitis: A Multicenter Study. PLoS One 4 (10): e0124172. https://doi.org/10.1371/journal.pone.0124172

6. Li Y, Poroyko V, Yan Z, et al (2016) Characterization of Intestinal Microbiomes of Hirschsprung's Disease Patients with or without Enterocolitis Using Illumina-MiSeq High-Throughput Sequencing. PLoS One 9 (11): e0162079. https://doi.org/10.1371/journal.pone.0162079

7. Plociennikowska A, Hromada-Judycka A, Borzecka K, Kwiatkowska K (2015) Co-operation of TLR4 and raft proteins in LPS-induced pro-inflammatory signaling. Cell Mol Life Sci 3 (72): 557-581. https://doi.org/10.1007/s00018-014-1762-5

8. Rosadini CV, Zanoni I, Odendall C, et al (2015) A Single Bacterial Immune Evasion Strategy Dismantles Both MyD88 and TRIF Signaling Pathways Downstream of TLR4. Cell Host Microbe 6 (18): 682-693. https://doi.org/10.1016/j.chom.2015.11.006

9. Leaphart CL, Cavallo J, Gribar SC, et al (2007) A critical role for TLR4 in the pathogenesis of necrotizing enterocolitis by modulating intestinal injury and repair. J Immunol 7 (179): 4808-4820. https://doi.org/10.4049/jimmunol.179.7.4808

10. Liu S, Gallo DJ, Green AM, et al (2002) Role of toll-like receptors in changes in gene expression and NF-kappa B activation in mouse hepatocytes stimulated with lipopolysaccharide. Infect Immun 7 (70): 3433-3442. https://doi.org/10.1128/iai.70.7.3433-3442.2002

11. Gibson DL, Ma C, Rosenberger CM, et al (2008) Toll-like receptor 2 plays a critical role in maintaining mucosal integrity during Citrobacter rodentium-induced colitis. Cell Microbiol 2 (10): 388-403. https://doi.org/10.1111/j.1462-5822.2007.01052.x 
12. Zhang J, Zheng Y, Luo Y, Du Y, Zhang X, Fu J (2019) Curcumin inhibits LPS-induced neuroinflammation by promoting microglial M2 polarization via TREM2/ TLR4/ NF-KB pathways in BV2 cells. Molecular Immunology (116): 29-37. https://doi.org/10.1016/j.molimm.2019.09.020

13. Frykman PK, Cheng Z, Wang X, Dhall D (2015) Enterocolitis causes profound lymphoid depletion in endothelin receptor B- and endothelin 3-null mouse models of Hirschsprung-associated enterocolitis. Eur J Immunol 3 (45): 807-817. https://doi.org/10.1002/eji.201444737

14. Porokuokka LL, Virtanen HT, Lindén J, et al (2019) Gfra1 Underexpression Causes Hirschsprung's Disease and Associated Enterocolitis in Mice. Cellular and Molecular Gastroenterology and Hepatology 3 (7): 655-678. https://doi.org/10.1016/j.jcmgh.2018.12.007

15. Mao YZ, Tang ST, Li S (2018) Duhamel operation vs. transanal endorectal pull-through procedure for Hirschsprung disease: A systematic review and meta-analysis. J Pediatr Surg 9 (53): 1710-1715. https://doi.org/10.1016/j.jpedsurg.2017.10.047

16. Dore M, Vilanova Sanchez A, Triana Junco P, et al (2019) Reliability of the Hirschsprung-Associated Enterocolitis Score in Clinical Practice. Eur J Pediatr Surg 1 (29): 132-137. https://doi.org/10.1055/s0038-1677046

17. Tang WB, Su Y, Yuan C, et al (2020) Prospective study reveals a microbiome signature that predicts the occurrence of post-operative enterocolitis in Hirschsprung disease (HSCR) patients. Gut Microbes 4 (11): https://doi.org/10.1080/19490976.2020.1711685

18. Zhang X, Li L, Li SL, Li SX, Wang XY, Tang ST (2020) Primary laparoscopic endorectal pull-through procedure with or without a postoperative rectal tube for hirschsprung disease: a multicenter perspective study. Journal of Pediatric Surgery 3 (55): 381-386.

https://doi.org/10.1016/j.jpedsurg.2019.06.013

19. Pruitt LCC, Skarda DE, Rollins MD, Bucher BT (2020) Hirschsprung-associated enterocolitis in children treated at US children's hospitals. J Pediatr Surg 3 (55): 535-540. https://doi.org/10.1016/j.jpedsurg.2019.10.060

20. Taylor MA, Bucher BT, Reeder RW, et al (2020) Comparison of Hirschsprung Disease Characteristics between Those with a History of Postoperative Enterocolitis and Those without: Results from the Pediatric Colorectal and Pelvic Learning Consortium. European Journal of Pediatric Surgery https://doi.org/10.1055/s-0040-1716876

21. Cheng Z, Zhao L, Dhall D, Ruegger PM, Borneman J, Frykman PK (2018) Bacterial Microbiome Dynamics in Post Pull-Through Hirschsprung-Associated Enterocolitis (HAEC): An Experimental Study Employing the Endothelin Receptor B-Null Mouse Model. Front Surg (5): 30. https://doi.org/10.3389/fsurg.2018.00030

22. Halleran DR, Ahmad H, Maloof E, et al (2020) Does Hirschsprung-Associated Enterocolitis Differ in Children With and Without Down Syndrome? J Surg Res (245): 564-568. https://doi.org/10.1016/j.jss.2019.06.086

23. Singer G, Kashofer K, Castellani C, Till H (2018) Hirschsprung's Associated Enterocolitis (HAEC) Personalized Treatment with Probiotics Based on Gene Sequencing Analysis of the Fecal 
Microbiome. Case Rep Pediatr (2018): 3292309. https://doi.org/10.1155/2018/3292309

24. Jiao CL, Chen XY, Feng JX (2016) Novel Insights into the Pathogenesis of Hirschsprung's-associated Enterocolitis. Chin Med J (Engl) 12 (129): 1491-1497. https://doi.org/10.4103/0366-6999.183433

25. Prato AP, Bartow-McKenney C, Hudspeth K, et al (2019) A Metagenomics Study on Hirschsprung's Disease Associated Enterocolitis: Biodiversity and Gut Microbial Homeostasis Depend on Resection Length and Patient's Clinical History. Frontiers in Pediatrics (7): https://doi.org/ARTN 326 $10.3389 /$ fped.2019.00326

26. Cheng Z, Wang X, Dhall D, et al (2011) Splenic lymphopenia in the endothelin receptor B-null mouse: implications for Hirschsprung associated enterocolitis. Pediatr Surg Int 2 (27): 145-150. https://doi.org/10.1007/s00383-010-2787-y

27. Gosain A, Frykman PK, Cowles RA, et al (2017) Guidelines for the diagnosis and management of Hirschsprung-associated enterocolitis. Pediatr Surg Int 5 (33): 517-521. https://doi.org/10.1007/s00383-017-4065-8

28. Mattar AF, Coran AG, Teitelbaum DH (2003) MUC-2 mucin production in Hirschsprung's disease: possible association with enterocolitis development. J Pediatr Surg 3 (38): 417-421; discussion 417421. https://doi.org/10.1053/jpsu.2003.50071

29. Yildiz HM, Carlson TL, Goldstein AM, Carrier RL (2015) Mucus Barriers to Microparticles and Microbes are Altered in Hirschsprung's Disease. Macromol Biosci 5 (15): 712-718. https://doi.org/10.1002/mabi.201400473

30. Song H, Zhang J, He W, Wang P, Wang F (2019) Activation of Cofilin Increases Intestinal Permeability via Depolymerization of F-Actin During Hypoxia in vitro. Front Physiol (10): 1455. https://doi.org/10.3389/fphys.2019.01455

31. Ye X, Sun M (2017) AGR2 ameliorates tumor necrosis factor-alpha-induced epithelial barrier dysfunction via suppression of NF-kappaB p65-mediated MLCK/p-MLC pathway activation. Int J Mol Med 5 (39): 1206-1214. https://doi.org/10.3892/ijmm.2017.2928

32. Tan Y, Zanoni I, Cullen TW, Goodman AL, Kagan JC (2015) Mechanisms of Toll-like Receptor 4 Endocytosis Reveal a Common Immune-Evasion Strategy Used by Pathogenic and Commensal Bacteria. Immunity 5 (43): 909-922. https://doi.org/10.1016/j.immuni.2015.10.008

33. Wang W, Weng J, Yu L, Huang Q, Jiang Y, Guo X (2018) Role of TLR4-p38 MAPK-Hsp27 signal pathway in LPS-induced pulmonary epithelial hyperpermeability. BMC Pulm Med 1 (18): 178. https://doi.org/10.1186/s12890-018-0735-0

34. Yang D, Li S, Duan X, et al (2020) TLR4 induced Wnt3a-Dvl3 restrains the intensity of inflammation and protects against endotoxin-driven organ failure through GSK3 $\beta / \beta$-catenin signaling. Molecular Immunology (118): 153-164. https://doi.org/10.1016/j.molimm.2019.12.013

35. Wittmann A, Bron PA, van S, II, et al (2015) TLR signaling-induced CD103-expressing cells protect against intestinal inflammation. Inflamm Bowel Dis 3 (21): 507-519. https://doi.org/10.1097/MIB.0000000000000292 
36. Gibson DL, Montero M, Ropeleski MJ, et al (2010) Interleukin-11 reduces TLR4-induced colitis in TLR2-deficient mice and restores intestinal STAT3 signaling. Gastroenterology 4 (139): 1277-1288. https://doi.org/10.1053/j.gastro.2010.06.057

37. Zhang Y, Lu Y, Ma L, et al (2014) Activation of vascular endothelial growth factor receptor-3 in macrophages restrains TLR4-NF-kappaB signaling and protects against endotoxin shock. Immunity 4 (40): 501-514. https://doi.org/10.1016/j.immuni.2014.01.013

\section{Figures}

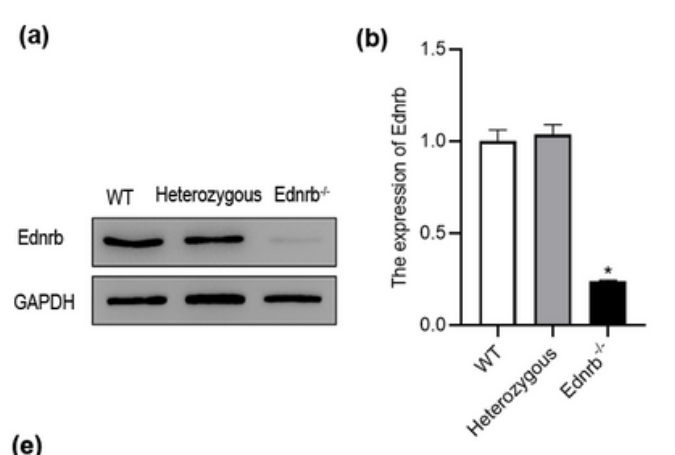

(e)
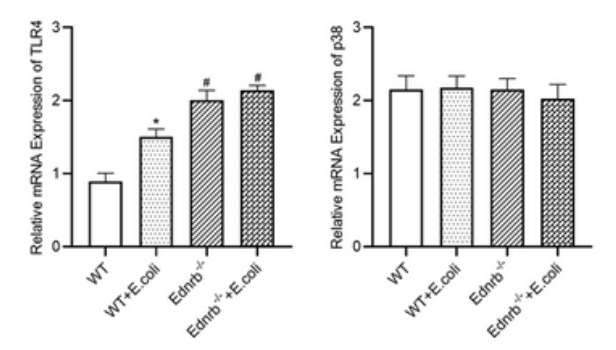
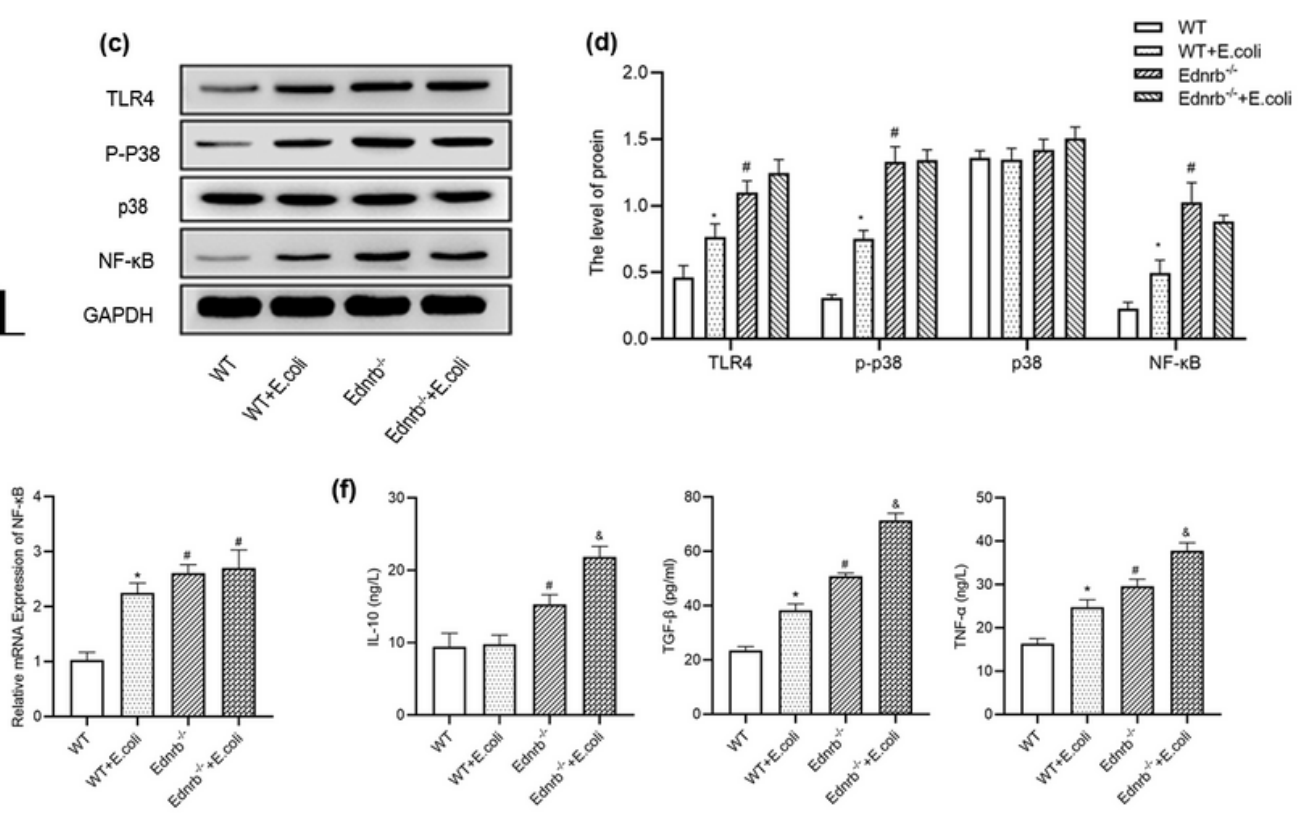

\section{Figure 1}

Identification of endothelin receptor B gene knockout $(a, b)$. The extracted proteins from the tails of mice subjected to western blot, and the following results were obtained: the Ednrb protein in Ednrb-/- mice was significantly decreased than WT and Heterozygous mice. (c, d) Western blot analysis of TLR4, p-p38, p38 and NF-KB protein expression levels in colonic tissues from four groups ( $n=5 /$ group). (e) mRNA expression of TLR4, p38 and NF-KB in colonic tissues from four groups. (f) Secreted TNF-a, TGF- $\beta$ and IL10 levels in colonic tissues from four groups. ${ }^{*} \mathrm{P}<0.05$ compared with WT group; \#P<0.05 compared with WT and E. coli-infected WT groups; \&P<0.05 compared with Ednrb-/-group, ( $n=5 /$ group). Data in (c), (b), (e) and (f) are normally distributed and expressed as the mean \pm SD. 


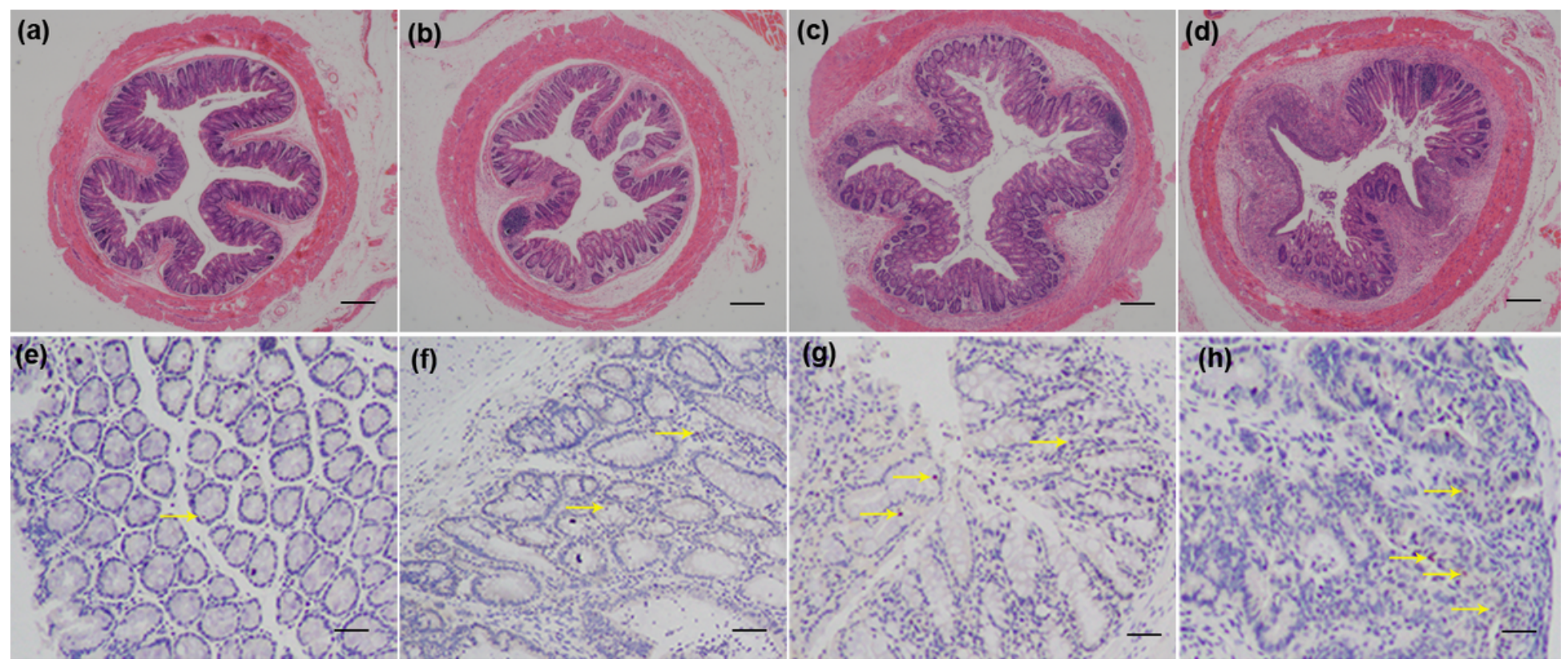

\section{Figure 2}

HE staining images (Version QC200; Nikon Corporation, Japan, magnification, x20) of colonic sections in four groups of mice. (a) WT group; (b) E. coli-infected WT group; (c)Ednrb-/- group; (d) E. coli-infected Ednrb-/- group. $(n=5 /$ group; Scale bar $=100 \mu \mathrm{m})$.Representative TLR4 immunohistochemical staining brown images (Version Olympus BH-2; Olympus Corporation, Japan, magnification, $\mathrm{x} 100$ ) of colonic sections (arrowheads)from (e) WT group; (f) E. coli-infected WT group; (g)Ednrb-/-group; (h) E. coliinfected Ednrb-/- group. (n=5/group; scale bar = $200 \mu \mathrm{m})$. 


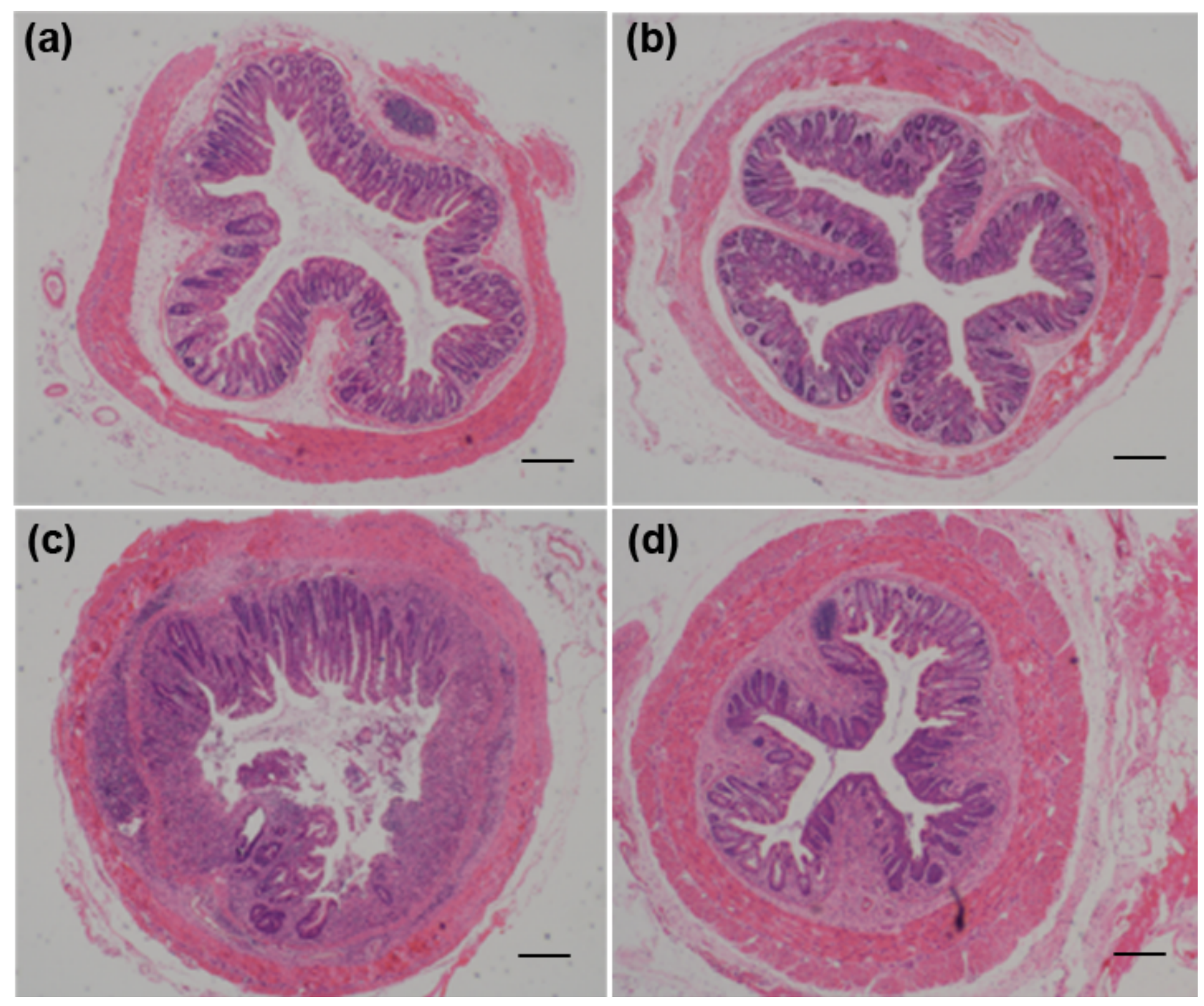

\section{Figure 3}

HE staining images (Version QC200; Nikon Corporation, Japan, magnification, x20) of colonic sections in four groups of TLR4 knockdown. (a) WT + E. coli group; (b) TLR4 siRNA-transfected WT + E. coli group; (c) Ednrb-/- + E. coli group; (d) TLR4 siRNA-transfected Ednrb-/- + E. coli group. ( $n=5 /$ group; Scale bar = $100 \mu \mathrm{m})$. 
(a)
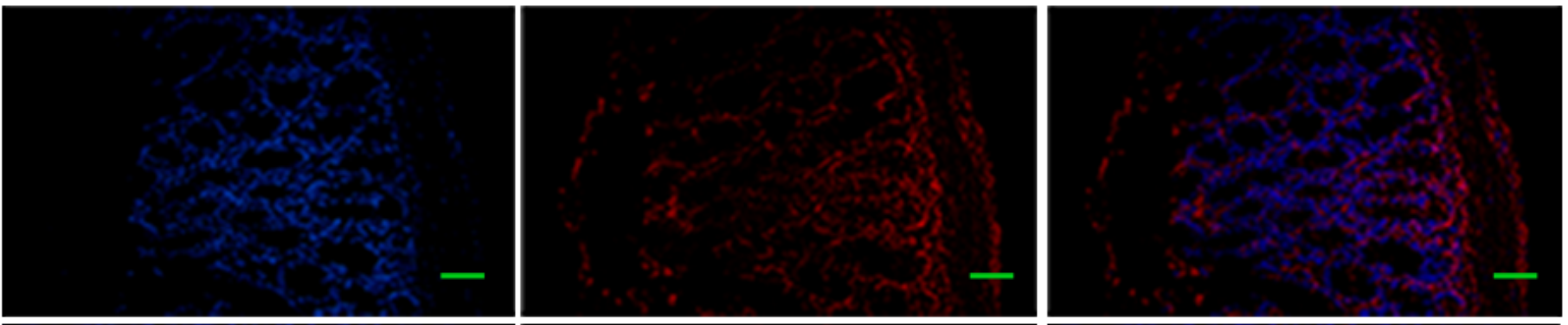

(b)
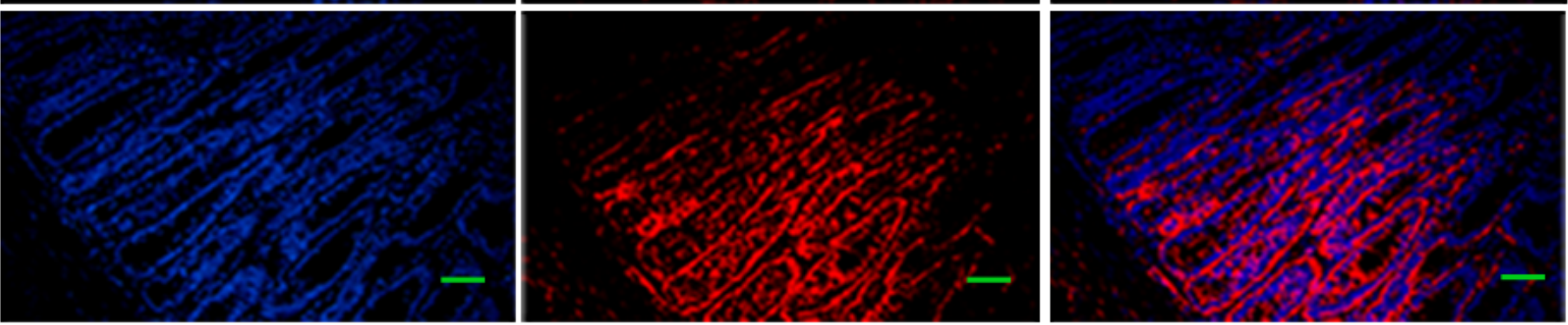

(c)
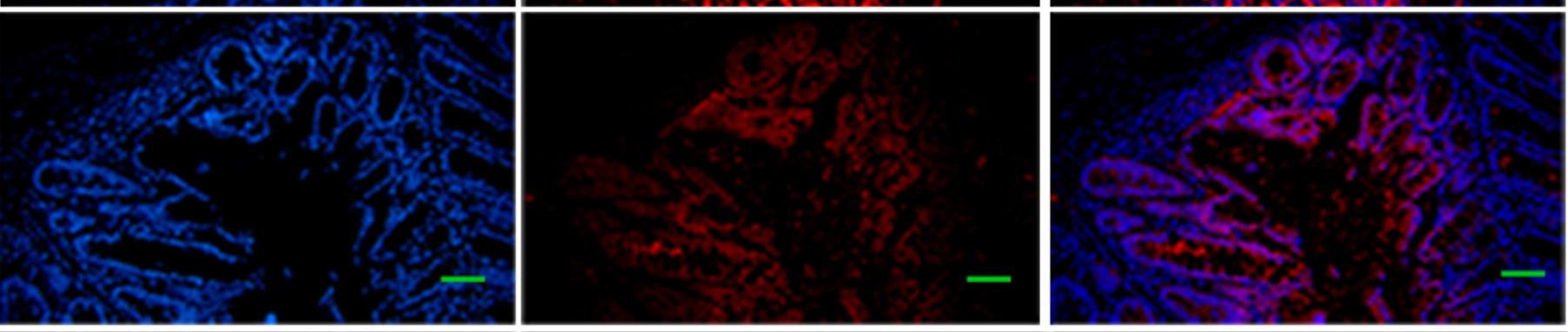

(d)
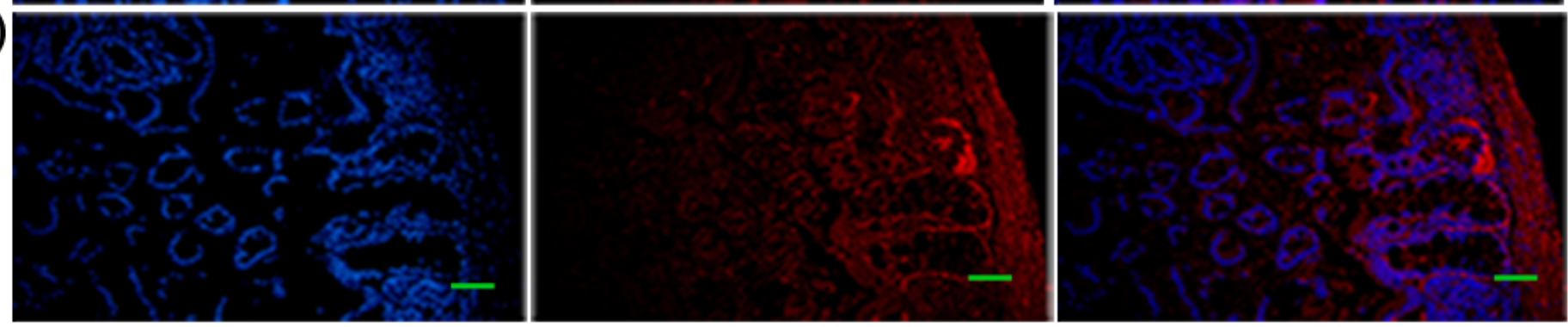

Figure 4

Representative F-actin immunofluorescence staining images (Version CKX41; Olympus Corporation, Japan, magnification, $x 20$ ) of colonic sections from (a) WT + E. coli group; (b) TLR4 siRNA-transfected WT + E. coli group; (c) Ednrb-/- + E. coli group; (d) TLR4 siRNA-transfected Ednrb-/- + E. coli group. $(n=5 /$ group; scale bar $=200 \mu \mathrm{m})$. 


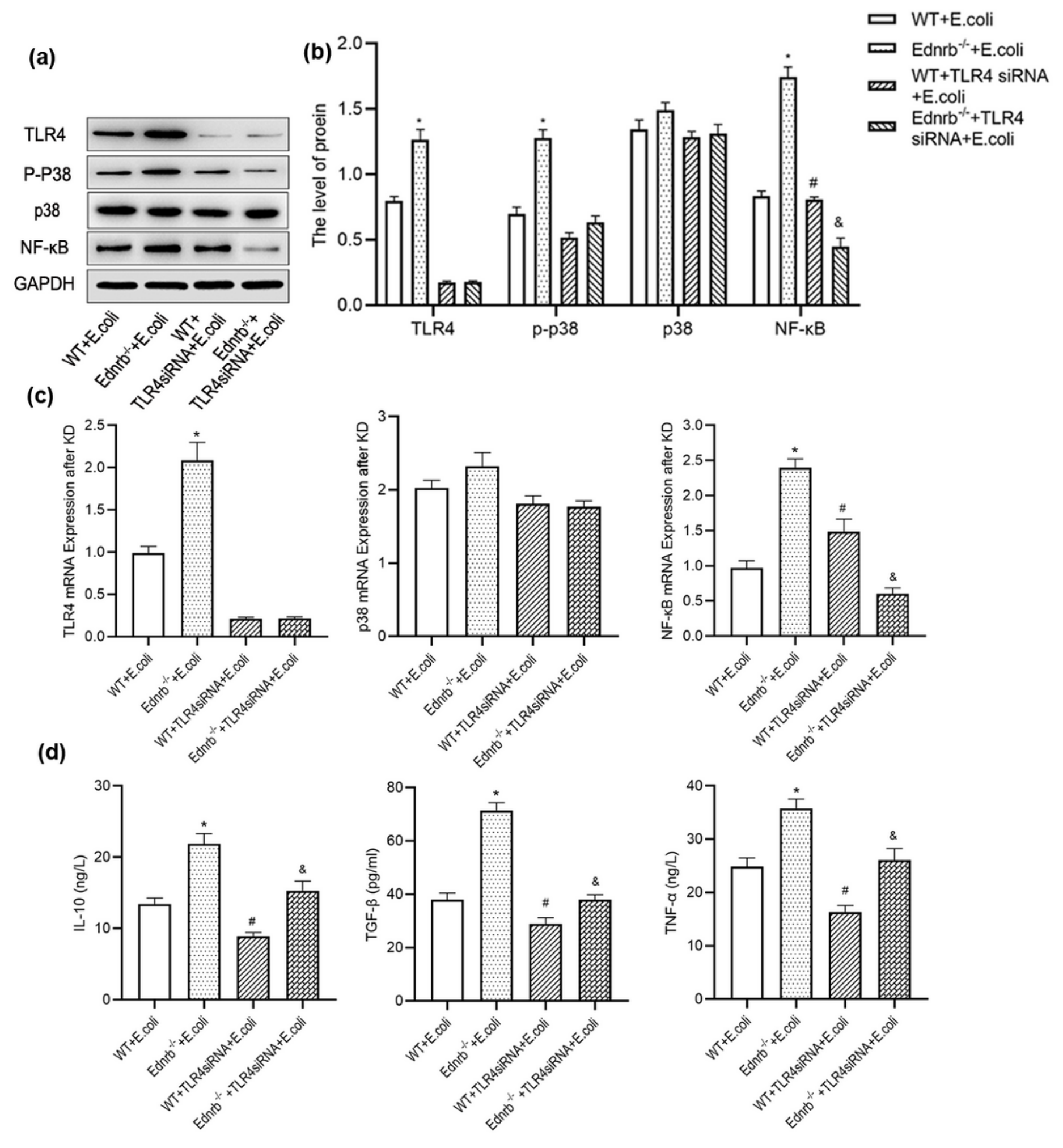

Figure 5

$(a, b)$ Western blot analysis of TLR4, p-p38, p38 and NF-KB protein expression levels in colonic tissues from four groups. (c) mRNA expression of TLR4, p38 and NF-KB in colonic tissues from four groups. (d) Secreted TNF-a, TGF- $\beta$ and IL-10 expression in colonic tissues from four groups. * $P<0.05$ compared with WT + E. coli, Ednrb-/- + E. coli and TLR4 siRNA-transfected Ednrb-/- + E. coli groups; \#P<0.05 compared with TLR4 siRNA-transfected Ednrb-/- + E. coli group; \&P<0.05 compared with WT + E. coli, TLR4 siRNA- 
transfected WT+ E. coli and Ednrb-/- + E. coli groups. ( $n=5 /$ group). Data in (b), (c) and (d) are normally distributed and expressed as the mean \pm SD. 